E

\title{
más maduro
}

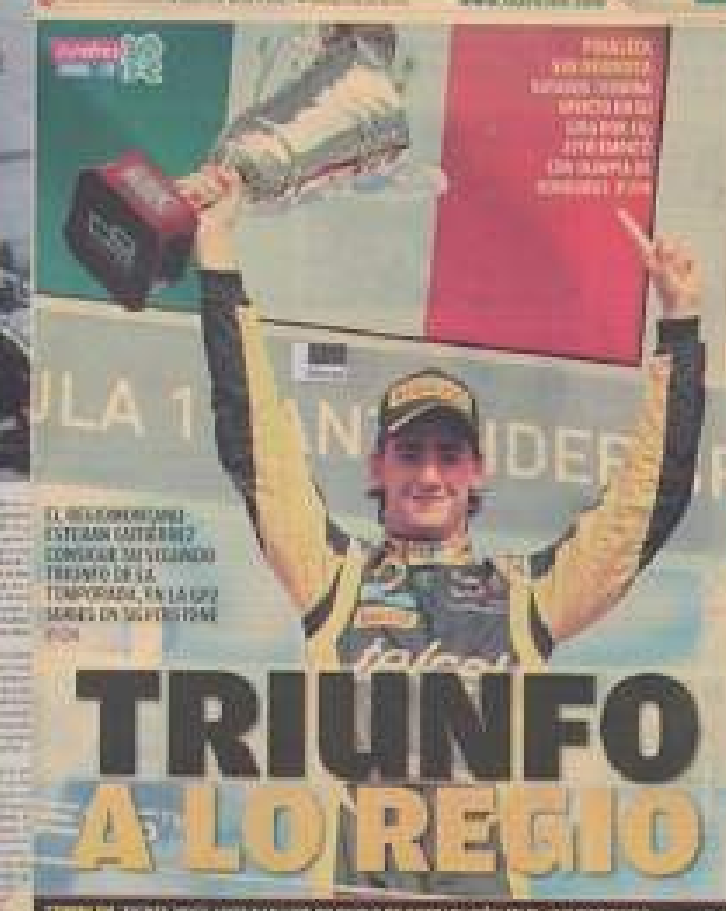

Gation 13

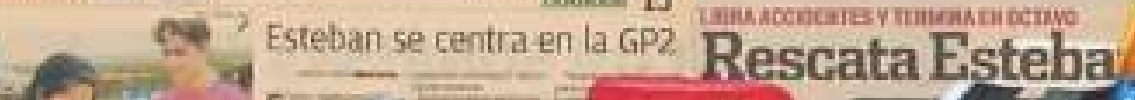
Kector
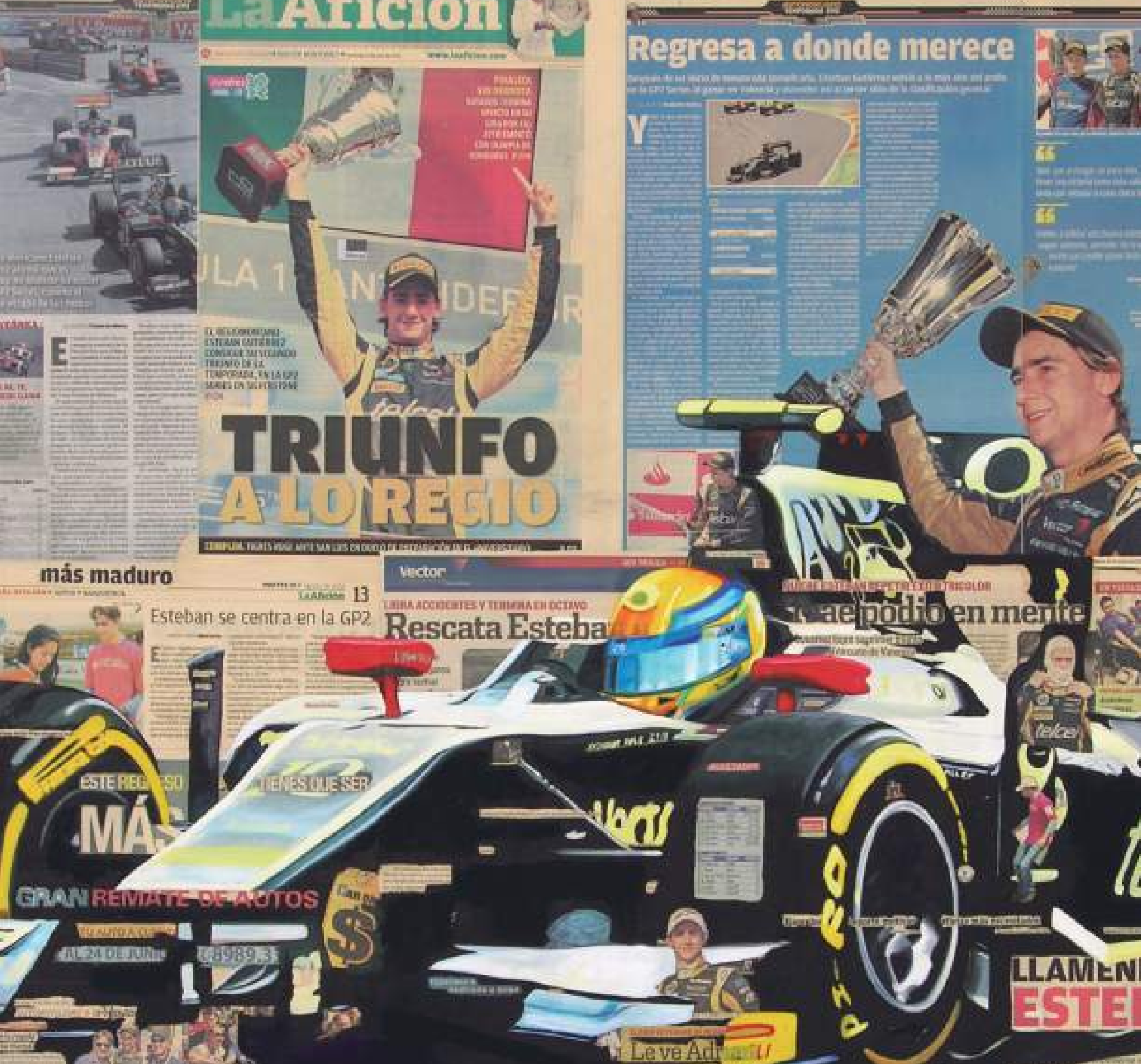

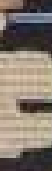
$\operatorname{lin}^{2} \frac{3}{6}$ 3 and

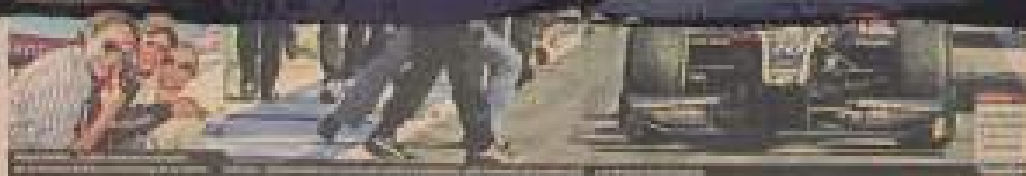

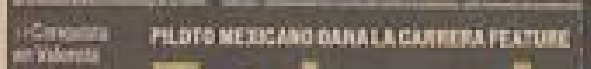
mint 2 in

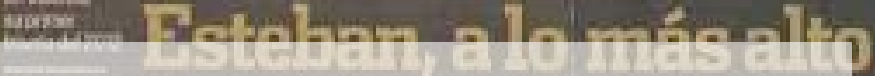

Título: "YA ES F1"

Autor: Salvador Díaz

Técnica: Óleo sobre Periódico

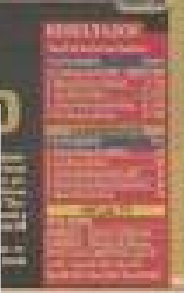

$\operatorname{man} 2 \ln =-$

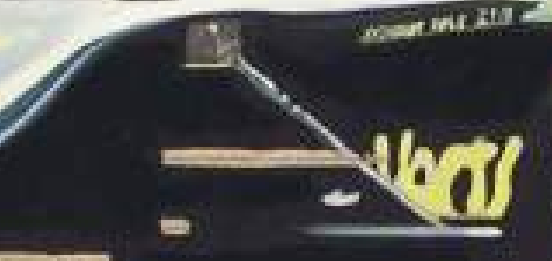

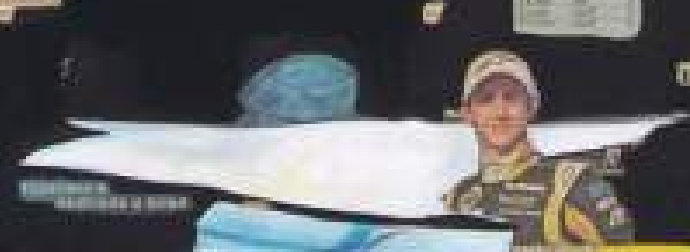

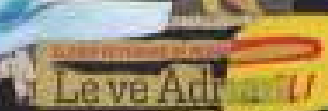
potencialde El?

is aepoodiben mente
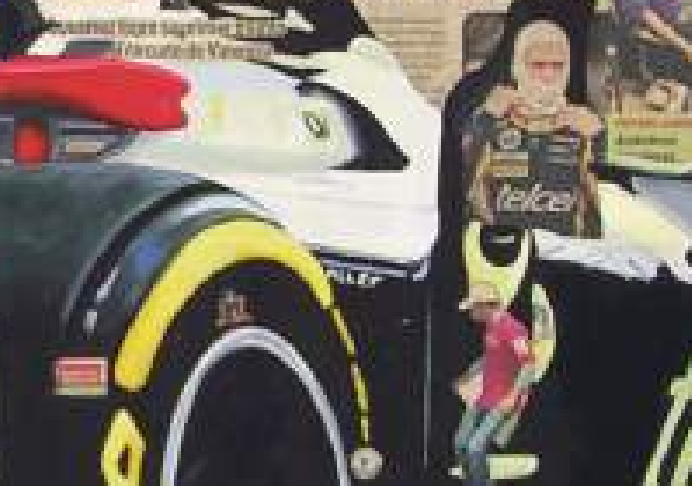


\section{PSICOLOGÍA Y MEDIACIÓN EN LA RESOLUCIÓN DE CONTROVERSIAS DEL DEPORTE UNIVERSITARIO}

\section{Psychology and mediation in the resolution of university sports controversies}

\section{JUAN MANUEL SÁNCHEZ LOZANO JOSÉ GUADALUPE STEELE GARZA}

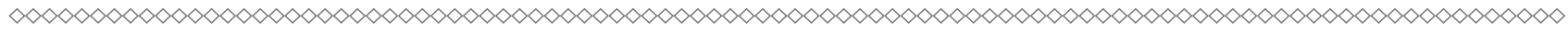

\section{RESUMEN}

La mediación es un método de resolución de conflictos en el que las partes llegan a acuerdos con la ayuda de un tercero neutral, imparcial y sin poder de decisión; es voluntaria, confidencial y está basada en el diálogo. El principal objetivo de la mediación deportiva es influir a todos los actores deportivos desde una perspectiva amplia sobre el conflicto deportivo y la mediación como herramienta indispensable para la resolución del mismo. Los estados de ánimo juegan un papel importante en el deporte, las emociones tanto en los entrenamientos, como en las competencias son determinantes en el rendimiento deportivo, uno de los deberes del mediador deportivo es trabajar con las emociones, conocer acerca del comportamiento emocional es vital para la gestión de los conflictos. El mediador cuenta con preparación y se ocupa en la intervención del conflicto, siendo un tercero imparcial, cuya función principal es ser un facilitador en la comunicación de los intervinientes en controversia, la finalidad es llegar a acuerdos. Ya que si las emociones de un deportista se encuentran en conflicto será más difícil alcanzar buenos resultados. El presente estudio trata de contribuir a la resolución de conflictos a través del mediador deportivo con las habilidades básicas de psicología actuando en sinergia con el objetivo de lograr el acuerdo consensuado de las partes.

PALABRAS CLAVE: mediación, deporte, psicología, conflicto, resolución y rendimiento.

\section{ABSTRACT}

Mediation is a method of conflict resolution in which the parties reach agreements with the help from a neutral, impartial third party, without decision-making power, is voluntary, confidential and is based on dialogue. The main objective of sports mediation is to influence all sports players from a broad perspective on sports conflict and mediation as an indispensable tool for the resolution of it. Moods play an important role in sport, emotions in both training and competitions are determinants in sports performance, one of the duties of a sports mediator is to work with emotions, knowing about emotional behavior is vital for the management of conflicts. The mediator has this preparation, is involved in the intervention of the conflict, as an impartial third party, having as its main function, to be a facilitator in the communication of the interveners who are in a dispute in order to reach an agreement. By this I mean that if the emotions of an athlete are in conflict will hardly achieve good results. The present study is to contribute to the resolution of conflicts through the sports mediator with the basic skills of psychology acting in synergy with the objective to achieve the consensus agreement of the parties.

KEYWORDS: mediation, sport, psychology, conflict, resolution and performance. 


\section{ANTECEDENTES}

os conflictos que ocurren en el contexto deportivo son muchos - y muy variados, desde el deporte amateur, alto rendimiento y profesional pudiendo suceder éstos en juegos olímpicos, juegos panamericanos 0 bien a nivel nacional en las olimpiadas nacionales y a nivel estudiantil en universiadas nacionales. Existen conflictos deportivos entre los diversos actores del mundo del deporte como: entrenadores, jugadores, árbitros, directiva, afición, medios, entre otros.

El presente trabajo trata de demostrar que las emociones están inmersas en la práctica deportiva y que cuando éstas se encuentran en un estado alterado, impiden al deportista realizar su mejor desempeño deportivo ya sea por problemas personales con su entrenador, con algún compañero de equipo, entre otros. Los deportistas en conflicto con sus entrenadores, se comprometen emocionalmente mermando la paz hacia el interior de los equipos y afectando su rendimiento deportivo.

La ansiedad y concentración son variables psicológicas implicadas en la esfera emocional del deportista y si no se encuentran estables, los resultados deportivos no serán los esperados. En este caso el mediador deportivo con las habilidades básicas de la ciencia de la psicología aplicada al deporte, logrará una sinergia al complementar ambos conocimientos en la resolución de disputas. Es fundamental conocer del funcionamiento de las emociones para la adecuada gestión de los conflictos. Un psicólogo posee competencias y habilidades para desempeñarse como un posible mediador con las características del conocimiento acerca de la conducta humana en su contexto emocional (Bernal, 2006).

La figura del psicólogo en función mediadora, posibilita soluciones satisfactorias en un procedimiento de mediación, por la simple razón que éste cuenta con sus métodos y técnicas de intervención psicológica, sobre todo en la esfera emocional que rodea al conflicto. En su rol de mediador percibe desde un principio aspectos actitudinales de riesgo que afectan de manera negativa la interacción y comunicación de los intervinientes.

En este sentido, la implicación del mediador deportivo, permite sustraer la carga emotiva de un deportista en conflicto ya que, al explorar su estado emocional, posibilita el conocer los antecedentes y eventos que forman parte de la disputa y considerar acciones convenientes que nos encaminen a un buen desarrollo de la sesión de mediación. Brindar contención emocional puesto que en una mediación aparecen estados de ánimo y actitudes diversas que tendrán que controlarse para la buena marcha del procedimiento de mediación.
La psicología del deporte es una ciencia que estudia las emociones y el comportamiento humano en el deporte y se ha venido desarrollando en el campo de conocimiento científico logrando consolidarse al paso del tiempo. La Comisión de Apelación y Arbitraje del Deporte (MASC) realiza procedimientos que buscan la solución a los conflictos entre las partes, ya sea de manera directa entre ellas (o con el nombramiento de agentes negociadores, como es el caso de la negociación) o mediante la intervención de un tercero imparcial (como son los casos de la mediación, la conciliación y el arbitraje).

En el mundo del deporte existe una gran cantidad de relaciones interpersonales, entre deportistas, entrenadores, técnicos, las federaciones, los clubes, que tienen que trabajar colaborando los unos con los otros. Se requiere de rapidez y flexibilidad para solucionar las controversias que puedan surgir y es justo lo que ofrece la mediación.

Los derechos de retransmisión de un partido, las diferencias surgidas en un contrato de un jugador, los conflictos entre clubes, entre otros, no pueden esperar a los tiempos implantados en la vía jurisdiccional para resolver di-

\section{La Comisión de Apelación} y Arbitraje del Deporte (MASC) realiza procedimientos que buscan la solución a los conflictos entre las partes, ya sea de manera directa entre ellas o mediante la intervención de un

tercero imparcial. 


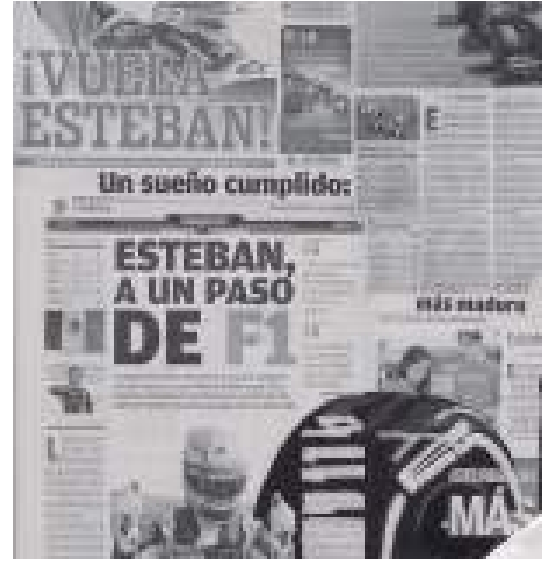

cho conflicto, ya que los calendarios deportivos no esperan.

Muchos son los conflictos emocionales de un deportista y su relación con su entrenador, puesto que pasan muchas horas de entrenamiento. Es pertinente el estudio de variables psicológicas implicadas en el deporte, en el rendimiento deportivo el equilibrio emocional es importante, cuando un deportista no se encuentra emocionalmente estable difícilmente logrará buenas marcas.

La implementación de una mediación deportiva como estrategia en la resolución de conflictos, brinda la oportunidad de que las partes asuman la responsabilidad en la gestión del conflicto, por otra parte. La mediación ofrece una pronta respuesta que permite amortiguar el efecto que pueda producir en la competencia en curso.

\section{OBJETIVO}

Demostrar que la mediación deportiva en la resolución de conflictos brindará aportes significativos en el rendimiento deportivo actuando en las emociones de los deportistas protagonistas de un conflicto. La hipótesis de este trabajo es que las emociones implicadas en el bajo
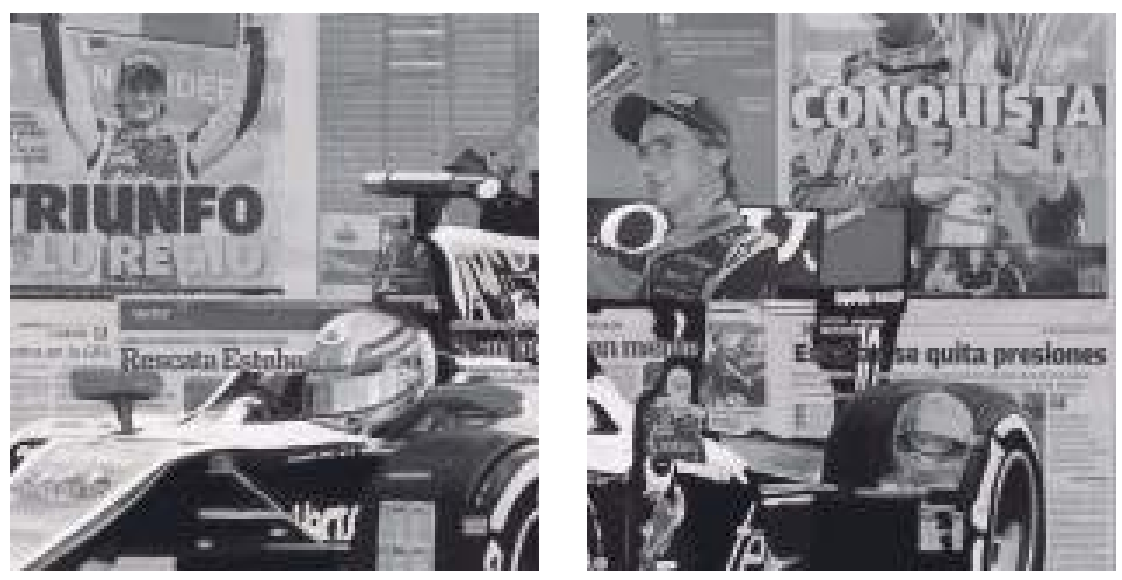

rendimiento deportivo de los atletas universitarios se podrían resolver a través de la mediación y la incidencia de las variables psicológicas: Ansiedad, Concentración y Motivación.

\section{METODOLOGÍA}

En la presente investigación se aplicó un cuestionario a una muestra de 39 deportistas universitarios de las siguientes disciplinas deportivas de los equipos Jaguares de la Universidad Regiomontana: Futbol Rápido Femenil, Futbol Soccer y Básquetbol Varonil con edades que oscilan entre 18 años a 21 años.

El instrumento básico utilizado en la investigación fue la encuesta mediante un cuestionario. Este instrumento de recolección de datos estandarizado operacionaliza las variables objeto de observación e investigación. El cuestionario comprende de 8 preguntas que corresponden a la variable Ansiedad, 8 preguntas que corresponden a la variable Concentración y 9 preguntas correspondientes a la variable Motivación.

Para la aplicación de la encuesta a los deportistas se llegó a un acuerdo con los entrenadores de las diferentes disciplinas deportivas; se les citó para explicar el objetivo de la misma, y se distribuyó el cuestionario entre los deportistas para su llenado. Una vez realizada la aplicación, los entrenadores ubicaron a los deportistas con los cuales existía confrontación, disputas y conflictos recurrentes, por lo que se les sugirió someterse a mediación con el objetivo principal de mejorar el rendimiento deportivo, haciendo énfasis de que debería tes y estar de acuerdo con el proceso, dicho lo anterior, los entrenadores hablaron con los deportistas en cuestión aceptando el procedimiento, siendo 3 deportistas de fútbol soccer varonil, 1 de básquetbol varonil y una de fútbol rápido femenil.

Posteriormente se citaron a 3 deportistas de fútbol soccer y su entrenador al Centro de Litigación y Mediación de la Facultad de Derecho y Criminología de la Universidad Autónoma de Nuevo León para llevar a efecto la mediación, logrando las partes llegar a un acuerdo y firmar el convenio. Por otra parte, el deportista de básquetbol y su entrenador, así como la deportista de fútbol rápido y su entrenador fueron citados posteriormente al Centro de Litigación y Mediación de la FacDyC donde también se logró llegar a un acuerdo y firmar el convenio. existir voluntariedad en ambas par- 


\section{RESULTADOS: GRÁFICA 1}

\section{CAMBIOS ESCALA DE ANSIEDAD}

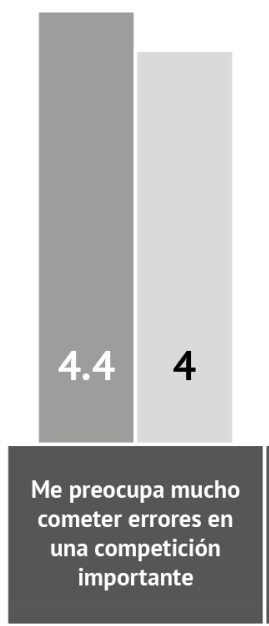

$2.8 \quad 2.4$

Cuando cometo un error en una competición me pongo muy ansioso
Media Pre

Media Post

En la Gráfica 1 se muestran los cambios reflejados en la aplicación previa y posterior a la medición donde se pueden observar los cambios sobresalientes en el aspecto de la ansiedad. Se observa un aumento en la tensión que genera el entrenador al deportista y se nota un descenso en la preocupación, ansiedad al equivocarse y tensión durante los entrenamientos, que se pueden deber a un efecto de la mediación que beneficia la confianza del sujeto.

\section{GRÁFICA 2 \\ GRAFICA 2}

CAMBIOS REGISTRADOS CONCENTRACIÓN

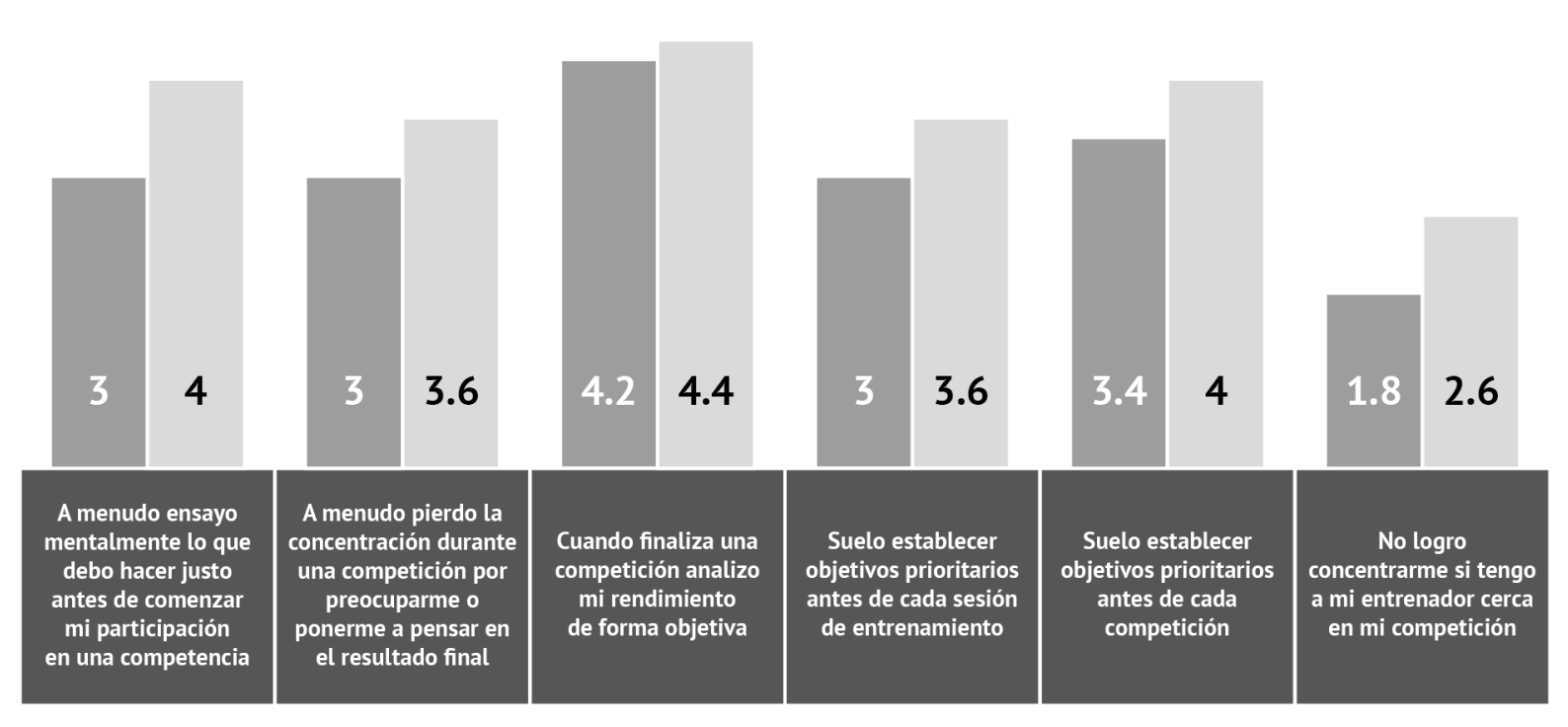

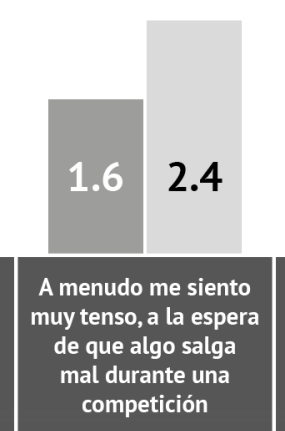
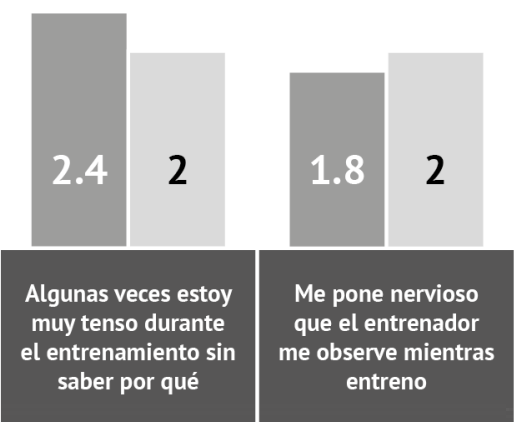

Media Post

En la Gráfica 2 se muestra el aspecto de la concentración, en la cual claramente se observan cambios los cuales son mayoritariamente positivos, siendo el cambio principal un aumento en la motivación propia, manifestado en mayor disposición para realizar preparación mental antes de los entrenamientos y las competencias. La mediación tiene como efecto secundario una mayor intervención de parte del entrenador. 


\section{GRÁFICA 3}

CAMBIOS REGISTRADOS MOTIVACIÓN

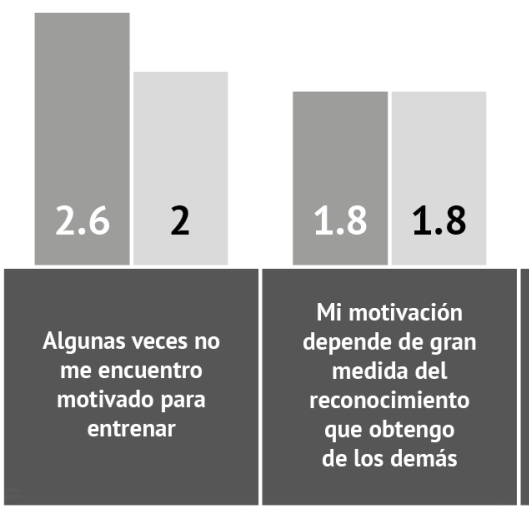

Media Pre

Media Post

La revisión de cambios en el aspecto motivacional, como se puede observar en la Gráfica 3, donde se indica que una intervención en mediación produce efectos positivos en la motivación para entrenar, un aumento de la dedicación del deportista con sus actividades, aumento de autoestima y conductas asertivas.

\section{CONCLUSIÓN}

Se considera a la mediación como una vía de gestión y resolución de conflictos en el ámbito deportivo y se ha planteado que la disminución del rendimiento de los atletas es consecuencia de situaciones conflictivas que influyen de manera negativa en la esfera emocional de los actores deportivos, ya que cuando un deportista no tiene control emocional debido a distractores externos en competencias o entrenamientos, difícilmente tendrá un buen desempeño en su deporte.

El contacto que se estableció con distintos coordinadores deportivos, entrenadores y deportistas definió el planteamiento de problema ya que mientras exista conflicto entrenador/ deportista, las emociones entran en juego corriendo el riesgo de ocasionar malas ejecuciones disminuyendo el rendimiento deportivo.

Promover la mediación como una cultura de paz en el ámbito de-

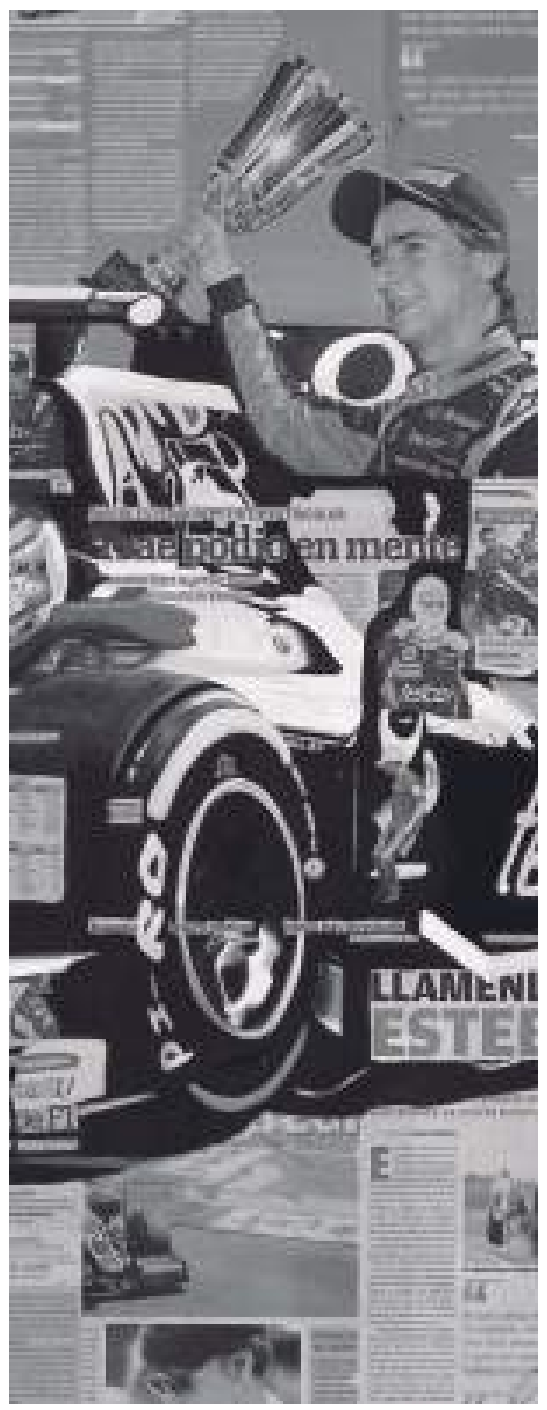

portivo, creando en las diferentes entidades del deporte una cultura de pacificación, con el fin de que sus miembros aprendan cómo resolver de manera independiente y pacífica sus disputas.

Es de suma importancia contar con la participación de las distintas instituciones deportivas desde sus categorías Amateur y Profesional, así como el deporte Estatal, Municipal y Universitario. Sería interesante poder incluir en los planes de estudio como asignatura de pregrado y posgrado, a la Mediación Deportiva en instituciones y dependencias académicas relacionadas con el deporte, en nuestro caso en la Facultad de Organización Deportiva de la Universidad Autónoma de Nuevo León. Por lo que proponemos implementar en las diferentes dependencias de nivel medio superior un departamento de mediación deportiva que permita solucionar las posibles controversias que se pudieran presentar en este contexto. 


\section{REFERENCIAS}

Bernal Samper, T. (2006) Psicólogo y mediación. Madrid.

Dosil, J. (2004). Psicología de la actividad física y deporte. Madrid, Mc Graw Hill. Buceta M, Larumbe E. El entrenador deportivo y la intervención psicológica, Texto, Experiencias en Psicología del deporte, Madrid, (2010), Edit. Dykinson. Garcia,N.V.A., Orlick, Terry, Entrenamiento mental, como vencer en el deporte y en la vida gracias al entrenamiento mental, Barcelona (2007), Paidotribo.

Gorjón, F.y Sánchez, A. (2015). Las 101 preguntas de la mediación. Guía práctica para el abogado. México: Editorial Tirant lo Blanch.

Gorjón, F. y Sánchez, A. (2016). Vademécum de mediación y arbitraje. México: Editorial Tirant lo Blanch.

Martín, M. (2015). Caracteres básicos y ventajas frente a otros sistemas de resolución de conflictos. En Mediación y deporte, Madrid, Dykinson. 


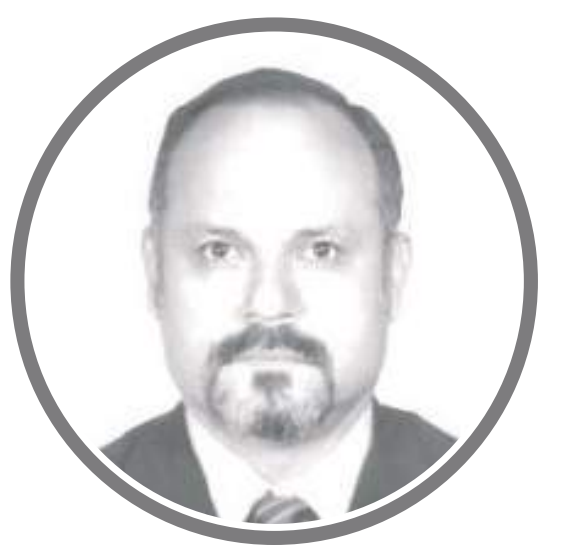

\section{Juan Manuel Sánchez Lozano}

Es Doctor en Métodos y Solución de Conflictos por la Facultad de Derecho y Ciencias Sociales UANL. Tiene Maestría en Formación y Capacitación de Recursos Humanos en la Facultad de Filosofía y Letras UANL y es Licenciado en Psicología en la Facultad de Psicología UANL. Cuenta con certificación internacional en programación neurolingüística en Quantum Leap Inc. y certificación en mediación por el Centro Estatal de Métodos Alternos para la Resolución de Conflictos. Su experiencia académica la ha desarrollado como Profesor de tiempo completo en la Preparatoria 25 UANL, como Coordinador del Centro de Mediación y Convivencia Escolar en la misma dependencia, entre otros cargos importantes dentro de la Universidad Autónoma de Nuevo León. Ha publicado diversos artículos en revistas arbitradas con temas como La psicología del deporte y su impacto en la resolución de conflictos a través de la mediación y La práctica de la Psicología del Deporte, entre el mito y la realidad, entre otros.

\section{Correo electrónico:}

psique08@hotmail.com

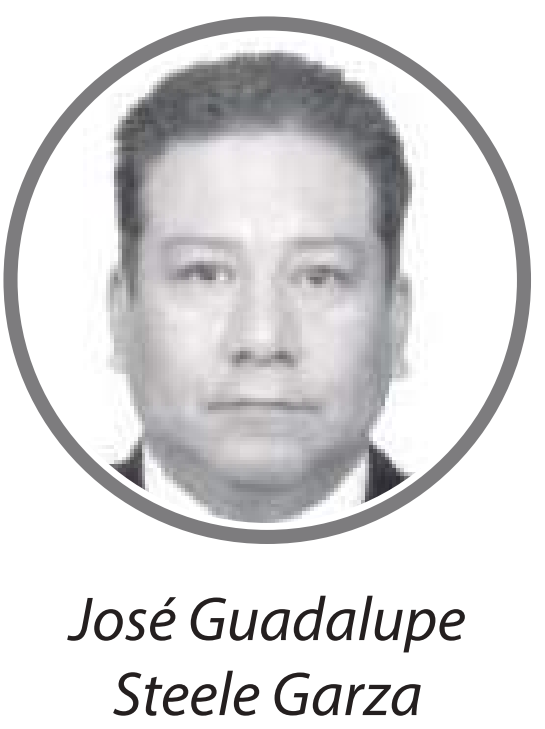

Es Doctor en Intervención y Mediación por la Universidad de Murcía, España con distinción Cum Laude. Maestría en Ciencias con la especialidad en Métodos Alternos de Solución de Controversias por la Facultad de Derecho y Criminología UANL. Su experiencia académica la ha desarrollado en diversos cargos importantes dentro de la Facultad de Derecho y Criminología UANL. Evaluador del Consejo Nacional para la Acreditación de la Educación Superior en Derecho A.C. (CONFEDE) y del Consejo Nacional de Ciencia y Tecnología (CONACYT). Ha sido conferencista y profesor invitado en universidades públicas y privadas. $\mathrm{Ha}$ colaborado con cargos importantes en diversas asociaciones como la Federación Nacional de Colegios Mediadores A.C., el Colegio de Abogados del Noreste, la Procuraduría Federal del Consumidor, entre otras. Es autor y coautor de 23 obras literarias a nivel local, nacional e internacional.

\section{Correo electrónico:}

psique08@hotmail.com

Recibido: 25/10/2018

Aceptado: 21/01/2019 